Article

\title{
A Modular Localization System as a Positioning Service for Road Transport
}

\author{
Peter Brida, Juraj Machaj * and Jozef Benikovsky \\ Department of Telecommunications and Multimedia, Faculty of Electrical Engineering, \\ University of Zilina, Univerzitna 1, Zilina 010 26, Slovakia; \\ E-Mails: peter.brida@fel.uniza.sk (P.B.); jbenikovsky@avasoft.sk (J.B.) \\ * Author to whom correspondence should be addressed; E-Mail: juraj.machaj@fel.uniza.sk; \\ Tel.: +42-141-513-2236; Fax: +42-141-513-1520.
}

External Editor: Felipe Jimenez

Received: 11 September 2014; in revised form: 6 October 2014 / Accepted: 14 October 2014 / Published: 28 October 2014

\begin{abstract}
In recent times smart devices have attracted a large number of users. Since many of these devices allow position estimation using Global Navigation Satellite Systems (GNSS) signals, a large number of location-based applications and services have emerged, especially in transport systems. However GNSS signals are affected by the environment and are not always present, especially in dense urban environment or indoors. In this work firstly a Modular Localization Algorithm is proposed to allow seamless switching between different positioning modules. This helps us develop a positioning system that is able to provide position estimates in both indoor and outdoor environments without any user interaction. Since the proposed system can run as a service on any smart device, it could allow users to navigate not only in outdoor environments, but also indoors, e.g., underground garages, tunnels etc. Secondly we present the proposal of a 2-phase map reduction algorithm which allows one to significantly reduce the complexity of position estimation processes in case that positioning is performed using a fingerprinting framework. The proposed 2-phase map reduction algorithm can also improve the accuracy of the position estimates by filtering out reference points that are far from the mobile device. Both algorithms were implemented into a positioning system and tested in real world conditions in both indoor and outdoor environments.
\end{abstract}


Keywords: localization; modular localization system; indoor positioning; hybrid positioning; seamless positioning

\section{Introduction}

In the recent years use of smart devices has increased significantly. Due to this, together with the fact that these devices have built-in Global Navigation Satellite Systems (GNSS) receivers, many location-based applications and services were created. The functions of many of these applications rely on the GNSS system because of its global availability. However these systems are highly reliable on Line of Sight (LoS) conditions, which are not the case in the urban environment. When the direct signal path between a transmitter (GNSS satellite) and a receiver (smart phone) is obstructed, the smart phone receives signals affected by multipath propagation and is not able to detect the direct signal. Another, even worse, condition is when the power of the received signal is lower than the minimum threshold at the GNSS receiver.

In both cases, localization accuracy can decrease significantly or in worst case the position of the device cannot be estimated at all. In the past decade many localization systems based on radio networks were proposed to deal with this problem and provide position estimates in environments where GNSS systems cannot be used, especially in indoor environments.

The most popular area for positioning systems and location based services (LBS) [1,2] is transport. With smart devices equipped with GNSS receivers, new possibilities for service providers were opened. Nowadays almost everyone owns a smart device and uses some basic LBS, like position estimation and navigation. However, based on previous analysis of the GNSS systems, these do not always provide position estimates which are accurate enough to provide good quality of service to the end user. To improve the performance of positioning systems, especially in areas where GNSS signal coverage is poor, alternative position estimates can be provided by positioning systems based on radio networks [3]. This can provide accurate position information even in indoor environments and thus could provide opportunities to provide new services e.g., navigation in underground parking lots and garages.

In this paper we will propose a modular positioning system, which at this time consists of three modules which can operate seamlessly in any environment covered with ubiquitously used radio networks, namely Global System for Mobile Communication (GSM) and Wireless Fidelity (Wi-Fi). The idea is to propose a positioning system that will be able to work in any environment from open outdoor areas, to dense urban areas and indoor environments. The main advantage of this system is the fact that there is no need to modify the smart phone device and no need to build up new infrastructure. The service provider only needs to set up a localization server, which will do the position estimation based on requests from the users. Another advantage is that it can be extended by adding new modules based on new technologies to provide more robust and reliable results, once the technology becomes available in the smart device.

The rest of the paper will be organized as follows: In Section 2 related work in the area of positioning in radio networks is described, while in Section 3 the proposed modular positioning system 
will be introduced. Section 4 will introduce testing scenarios and the achieved results will be shown and discussed in Section 5. Section 6 will conclude this paper and will provide some thoughts about the future developments of the proposed system.

\section{Positioning in Wireless Networks}

In this section related work in the area of positioning using wireless networks will be described. Many different positioning systems have been developed recently utilizing different wireless technologies, e.g., Ultra-wideband (UWB) [4], ZigBee [5], Bluetooth [6], Wi-Fi [7-11], GSM [12-15] etc. In the paper we will focus on positioning in Wi-Fi and GSM since these technologies are almost ubiquitously deployed in the urban and indoor environment, where GNSS positioning is not reliable enough.

\subsection{Positioning in GSM Networks}

Since the GSM network represents the basis of modern area-wide wireless communication infrastructure, this technology is ideal for use in wireless positioning. The basic method for positioning in the GSM network is Cell of Origin ( $\mathrm{CoO}$ ) where the position of a mobile device is given by the position of the Base Transceiver Station (BTS) with the highest signal power. Advanced positioning in the GSM networks can be performed based on three approaches:

- Distance-based positioning,

- $\quad$ Angle-based positioning,

- $\quad$ GSM fingerprinting.

In the distance-based positioning the basic assumption is that the positioning service provider knows the exact positions of the BTS in the radio network and their transmission powers. In such a case the distance between the BTS and a mobile device can be calculated from the measured Received Signal Strength (RSS) using a suitable signal propagation model [13].

On the other hand, in angle-based positioning the only required information is the position of the BTS. The resolution of the measured Angle of Arrival (AoA) depends on the antenna configuration [13]. This information can be further diluted due to Non-Line-of-Sight (NLoS) conditions and multipath propagation. Angle-based positioning together with Round Trip Time (RTT) measurements is commonly used in the Enhanced Cell of Origin (ECoO) [14] method, where position of the mobile device can be estimated within a given sector of area covered by the BTS.

The last and one of the most common ways to provide positioning service in GSM networks is to use a fingerprinting localization framework. In GSM fingerprinting [15] the position of a mobile device is estimated by comparison of measured RSS values from surrounding BTS with RSS values stored in the database at the localization server. A detailed description of the fingerprinting framework will be provided in the Section 2.3.

\subsection{Positioning in Wi-Fi Networks}

Nowadays the Wi-Fi network infrastructure is almost ubiquitous in dense urban areas and in indoor environments and all new smart devices have integrated Wi-Fi transmitters. This has led to the development of positioning systems based on Wi-Fi networks, mainly in indoor environments. 
In Wi-Fi networks, like GSM, positioning can be performed in different ways. Positioning systems based on Wi-Fi commonly utilize one of the following frameworks:

- Distance- or angle-based positioning (multilateration),

- Wi-Fi fingerprinting.

Other positioning techniques can also be used, e.g., Cell of Origin ( $\mathrm{CoO}$ ) and propagation modeling; however these methods are not very common [16]. Propagation modeling is actually a modification of the fingerprinting approach where a radio map database is created using propagation models. This helps to reduce the complexity of the calibration phase; however the accuracy of such a system is significantly lower.

Multilateration positioning basically utilizes estimation of the distance or angle between transmitter and receiver. In Wi-Fi networks AoA can be estimated only when Multiple-Input and Multiple-Output (MIMO) technology is used and LoS conditions are required [17]. Nowadays this is not the common case, therefore positioning using AoA cannot be implemented in Wi-Fi.

On the other hand distance between transmitter and receiver can be estimated by measuring RSS Time of Arrival (ToA) or RTT [18]. Both methods have their drawbacks when it comes to the accuracy of the distance estimate. In RTT measurements the accuracy is affected by latencies, which falsify outgoing and incoming time stamps [19]. Resulting delays may have typical variation of $5 \mu$ s which results in $1500 \mathrm{~m}$ error in the distance estimation. Another error in distance estimation can be caused by clock drift of the RTT observations [20]. When RSS is used to estimate the distance between transmitter and receiver, accuracy is given mainly by the propagation model used to compute the distance from RSS. Another challenge for RSS-based lateration is the high time-variability of signal strength caused by fading and multipath phenomena [21].

The most popular localization framework in Wi-Fi networks is empirical fingerprinting. Like in GSM networks, RSS from Access Points (APs) in the communication range is measured and used to estimate the position of mobile devices by comparison with the radio map database. The main advantage is that fingerprinting positioning seems to be more immune to multipath propagation phenomena. Another advantage in contrast to lateration positioning, is that there is no need to know the position of APs. It is also important to note that in Wi-Fi networks the RSS values are measured from beacon signals. Therefore RSS is not affected by the adaptive power regulation implemented in APs and device does not need to connect to the network. Thus APs from different providers and with different transmitting power settings can be used without any impact on the positioning system.

\subsection{Fingerprinting Localization Framework}

In this section fingerprinting positioning will be described in detail, since it is the most common and reliable positioning framework used in both GSM and Wi-Fi networks. Fingerprinting seems to achieve best accuracy in comparison to other positioning frameworks, especially in areas with strong multipath propagation and NLoS conditions. The operation of positioning systems based on fingerprinting frameworks can be divided into two phases - the calibration phase and the positioning phase [22]. 


\subsubsection{Calibration Phase}

The calibration phase, sometimes also called offline phase, must be performed before the deployment of the positioning service. It represents a necessary step in the fingerprinting localization framework. During this phase a radio map database is created and stored in the database at the localization server.

During the calibration phase the localization area is divided into small cells, where each cell is represented by a single reference point [9]. At each reference point RSS values from all APs within range are measured and stored in the radio map database, which is a collection of data vectors that can be described as:

$$
S_{j}=\left(\alpha_{1}, \ldots, \alpha_{N_{j}}, c_{j}, \theta_{j}\right) \quad j=1,2, \ldots, M
$$

where $N_{j}$ is the number of APs heard at the $j$-th reference point, $M$ is the number of reference points, $\alpha_{i}$ are RSS values, $c_{j}$ represent coordinates of $j$-th reference point and parameter vector $\theta_{j}$ can contain any additional information that may be used in the localization phase. The principle of radio map database creation is depicted in Figure 1.

Figure 1. Radio map principle.

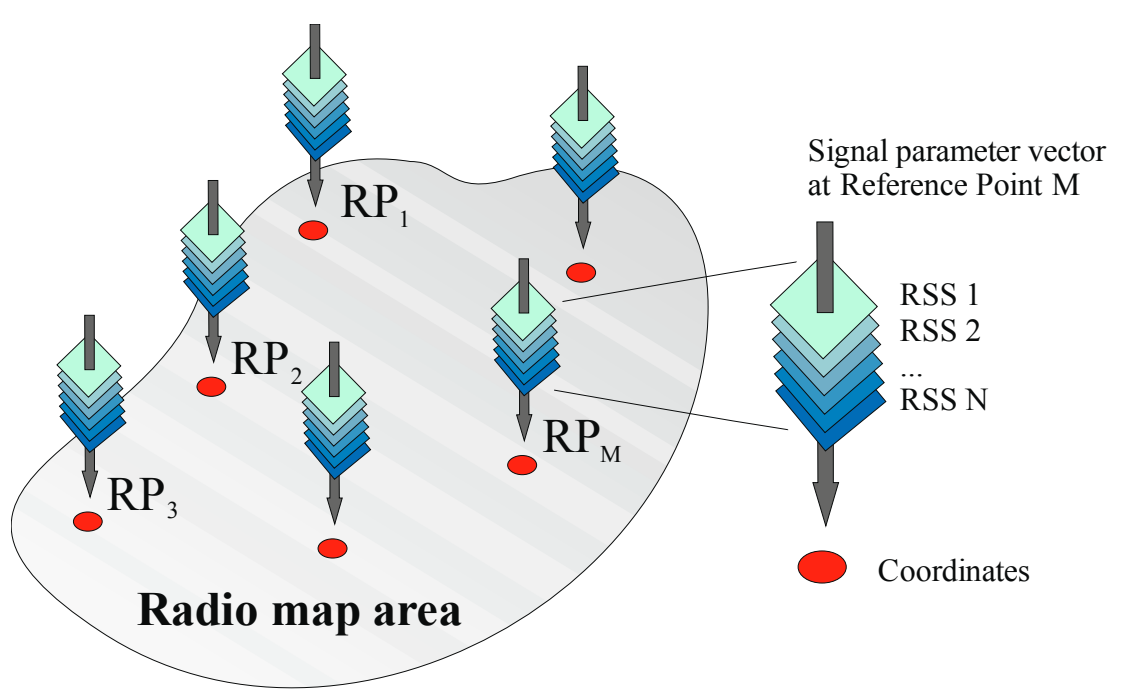

\subsubsection{Localization Phase}

During the localization phase, also called in some literature online phase, the position of the mobile device is estimated. The mobile device measures RSS values from all APs within range. These are sent to the localization server, which compares them to the data stored in the radio map database. Algorithms used for estimation of a position of the mobile device can be divided into two main groups-deterministic and probabilistic.

In the probabilistic (or statistical) framework the mobile device's position is modeled as a random vector. The location candidate $\gamma$ is chosen if its posterior probability is the highest [23]. The decision rule uses Bayes' theorem:

$$
P\left(c_{i} \mid S\right)=\frac{P\left(S \mid c_{i}\right) P\left(c_{i}\right)}{P(S)}
$$


where posterior probability $P\left(c_{i} \mid S\right)$ is a function of likelihood $P\left(S \mid c_{i}\right)$, prior probability $P\left(c_{i}\right)$ and observed evidence $P(S)=\sum P\left(S \mid c_{i}\right) P\left(c_{i}\right)$, vector $S$ represents the observed RSS values during online phase and $c_{i}$ stands for $i$-th location candidate, i.e., reference point (RP).

On the other hand deterministic algorithms are based on the assumption that RSS values at the receiver are not random and depend on the position of the mobile device [9]. Thus the position of a mobile device is estimated by searching for the highest similarity between the measurements from the device and the fingerprints stored in the radio map database. The position estimate is commonly computed using the estimator:

$$
\hat{x}=\frac{\sum_{i=1}^{M} \omega_{i} \cdot c_{i}}{\sum_{i=1}^{M} \omega_{i}}
$$

where $\omega_{i}$ is a non-negative weighting factor [9]. Weights can be calculated as the reciprocal of the distance between RSS vectors from the current measurements and radio map database. Usually the Euclidian distance is used, but different distance metrics are also possible [24].

The estimator of formula (3) that keeps the $K$ largest weights and sets the others to zero is called the Weighted K-Nearest Neighbours (WKNN) method [10]. WKNN with all weights $\omega_{i}=1$ is called the K-Nearest Neighbours (KNN) method. The simplest method, where $K=1$, is called the Nearest Neighbour (NN) method [11]. In [9] it was found that WKNN and KNN methods perform better than the NN method, particularly when values of parameter $K$ are 3 or 4.

In the literature it is shown that both probabilistic and deterministic algorithms are able to achieve similar results in terms of accuracy [23]. The advantage of the deterministic algorithms is that there is no need of an accurate statistical model to describe the signal characteristics in the environment. Therefore we decided to implement deterministic algorithms to the proposed modular positioning system.

\section{Proposed Algorithm and Modular System}

\subsection{Modular Localization System}

The proposed modular localization system is logically one level above the structure of standard localization systems. The components of a modular system have to be proposed according to the goals and requirements for such system.

The proposed system is an integrated set of components that provide localization services for its users. The proposed system should be able to provide services to multiple users and the service should be accessed simultaneously. The proposed localization system is centralized and thus a localization service is provisioned from one source called a localization server. It can be described as a network-based system with device assistance. This means that all computations are performed at the centralized localization server implemented on the network side of the system and the mobile device performs measurements when requested by the server. The system is fully autonomous, because it communicates using the existing infrastructure of telecommunications networks. Switching of the system mode (indoor or outdoor) is currently performed automatically based on data measured by mobile device. The process of switching is controlled by a modular localization algorithm (MLA). 
The proposed system is currently based on the assumption, that Global Positioning System (GPS), GSM and Wi-Fi together could allow ubiquitous positioning in an environment similar to that illustrated in Figure 2. Positioning modules based on these technologies are considered as basic localization modules of the system, since standard mobile phones contain the necessary hardware to exploit them. The system is open for implementation of other modules according to requirements, e.g., Bluetooth, Zig-Bee, etc.

Figure 2. Example of an environment.

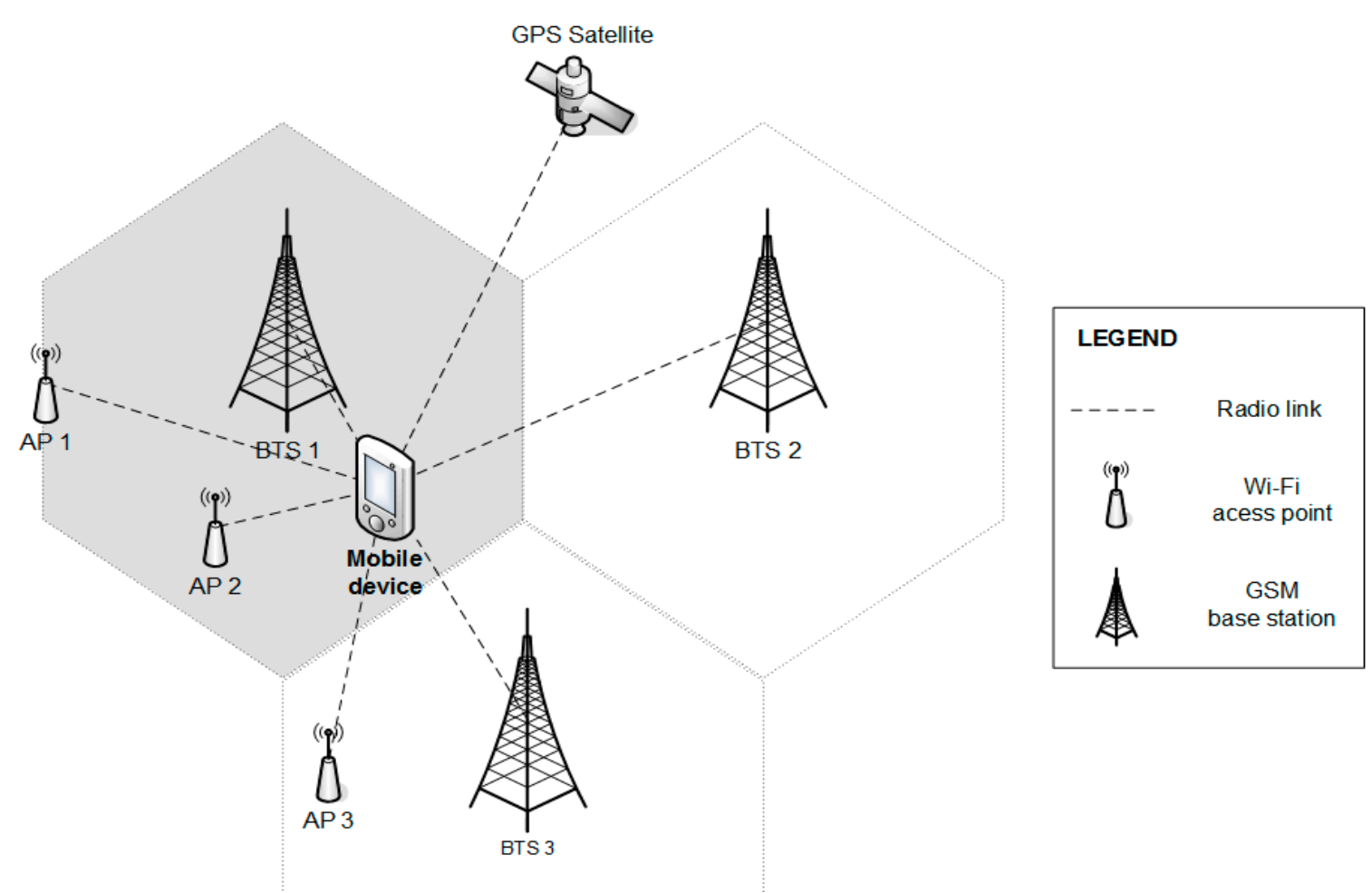

The system can be divided into several components that communicate with each other and have their own responsibilities. The basic components are mobile devices (localized terminals), existing network infrastructures and the localization server. In terms of architecture, it is possible to describe the system from multiple perspectives that explain the importance and role of individual components such as:

- functional view,

- component view,

- technological view,

- user view.

These perspectives will be presented in order to comprehensively describe the modular localization system implementation. The functional view also contains the description of the novel modular localization algorithm. 


\subsubsection{Functional View}

The functional view represents the role and responsibilities of the various components of the system. These tasks can be divided into multiple layers, namely:

- Presentation layer - Provides visualization of the position of the user, allows him to control the localization of software, make requests and import radio map data.

- Application logic layer-Contains algorithms for position estimation, data processing and validation, security control and user request processing.

- Service layer-Provides information transfer between individual components.

- Security layer - Prevents unauthorized access, secures the transmission of sensitive data.

- Management layer-Monitors important changes in the system, records the errors and allows their analysis.

Localization system features can be divided into these layers for the mobile station, as well for the localization server. This division is shown in Table 1.

Table 1. Responsibilities of components in the proposed modular localization system.

\begin{tabular}{|c|c|c|}
\hline Layer & Mobile Station & Localization Server \\
\hline Presentation & $\begin{array}{c}\text { Display user position } \\
\text { Display other relevant data } \\
\text { Enter localization request } \\
\text { Control of map data collection } \\
\text { Configuration of application }\end{array}$ & Display management interface \\
\hline Application logic & $\begin{array}{l}\text { Validation of input data } \\
\text { Measurement and processing of } \\
\text { localization data }\end{array}$ & $\begin{array}{l}\text { Validation of input data } \\
\text { Implementation of MLA and } \\
\text { partial localization algorithms } \\
\text { User request handling } \\
\text { Error handling }\end{array}$ \\
\hline Service & $\begin{array}{c}\text { Data exchange with localization } \\
\text { server } \\
\text { Localization service calls }\end{array}$ & $\begin{array}{l}\text { Data exchange with mobile } \\
\text { stations } \\
\text { Request transfer } \\
\text { Insertion of data into database } \\
\text { Reading of data from database }\end{array}$ \\
\hline Security & Securing of sensitive data & $\begin{array}{c}\text { Authentication } \\
\text { Authorization } \\
\text { Securing of sensitive data } \\
\end{array}$ \\
\hline Management & $\begin{array}{c}\text { Notify localization server about } \\
\text { errors }\end{array}$ & $\begin{array}{c}\text { Make record of errors } \\
\text { Make records of important changes } \\
\text { in the system }\end{array}$ \\
\hline
\end{tabular}

The individual layers are classified into three levels, where each layer communicates directly only with neighboring layers. Security and management layer are needed at every level and can communicate with any of the three layers. All layers in the system are shown in Figure 3. 
Figure 3. Functional layers in the modular localization system.

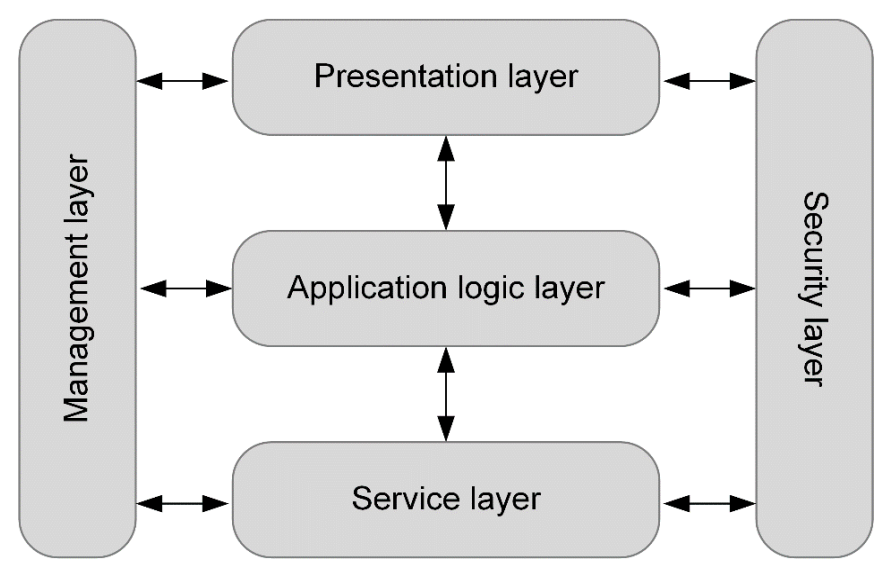

The application logic layer contains the original MLA algorithm. The algorithm is responsible for handling localization requests with all necessary signal information from GPS, GSM and Wi-Fi, selecting the most appropriate platform and returning the position. The details are depicted in the algorithm flowchart in Figure 4. The algorithm facilitates deterministic the fingerprinting localization framework, described in the previous section, together with the proposed map reduction algorithm.

Figure 4. Flowchart of modular localization algorithm.

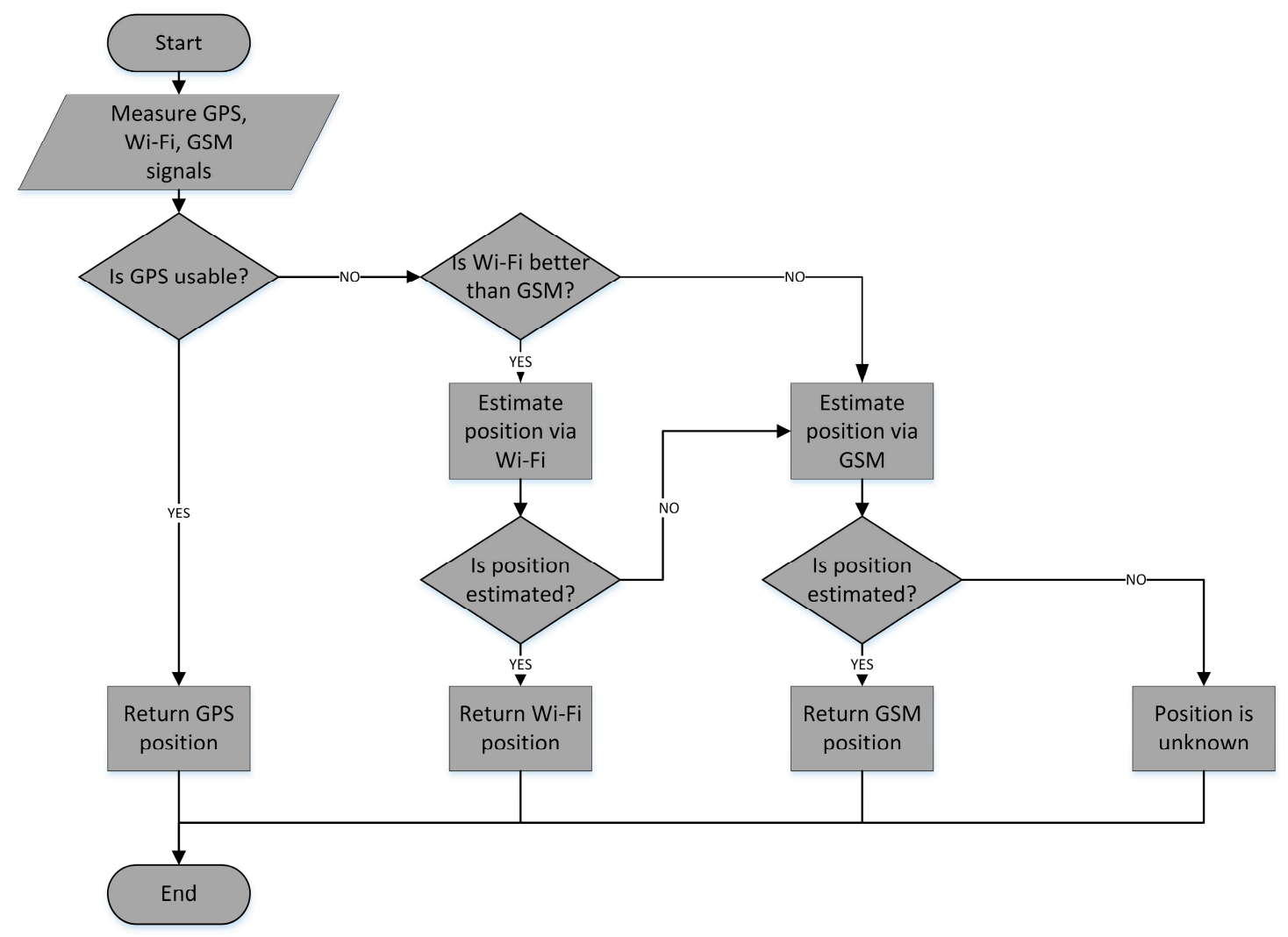

In short, the MLA checks GPS availability and if the position can be determined, returns the position to the mobile device. However, GPS may not be available in scenarios involving missing GPS hardware, not enough visible satellites or indoor environments. In that case Wi-Fi or GSM measurements are used. The number of base stations with RSS $10 \mathrm{dBm}$ above the minimum 
measurable signal level is calculated for the Wi-Fi network $\left(N_{A P}\right)$ and GSM network $\left(N_{B T S}\right)$. If $N_{A P}$ is greater than or equal to 3, Wi-Fi localization is selected. The threshold of three transmitters (APs or BTSs) was chosen, since signals from at least three transmitters are needed to clearly define position in 2D space. If that localization fails, e.g., similar radio map vectors are missing due to a non-mapped area or if $N_{A P}<3$, GSM localization is performed.

The RSS thresholds were set to $-90 \mathrm{dBm}$ for Wi-Fi signals and $-103 \mathrm{dBm}$ for GSM signals, since most devices provide minimum RSS values of $-100 \mathrm{dBm}$ and $-113 \mathrm{dBm}$ for Wi-Fi and GSM signals, respectively. This threshold was added based on previous results published in [25] which show that low RSS values have a negative impact on localization accuracy, caused by the fact that these values are more significantly affected by RSS fluctuations.

\subsubsection{Component View}

The modular view presents the components of the system in terms of unique characteristics and interdependencies. Modularity enables later improvements and addition of features to the system in the future. Individual modules can extend the functionality of mobile stations, localization server or a localization system as a whole. The proposed modules relate to localization system as a whole and their roles are as follows:

- GNSS module - Localization module that utilizes one or more GNSS, initially GPS.

- GSM module-Localization module that utilizes GSM network.

- Wi-Fi module-Localization module that utilizes Wi-Fi network.

- Communication module-Data exchange and requirement transfer between the mobile station and the localization server.

- Presentation module-Display position of a user on a suitable map of the environment, through which he can determine what is in its surroundings.

- Security module-Security of communication between the mobile station and the localization server.

- Application logic module-User or mobile station request processing.

Modules should be substitutable and thus, for example when safety requirements change, the security module can be substituted by a more suitable one or a new localization module such as WiMAX or Bluetooth can be added.

\subsubsection{Technological View}

The technological view provides an overview of the technologies and the standards by which the system is constructed. The list of proposed technologies for localization server and mobiles stations is given in Table 2. The list consists of technologies used in the system developed by the authors, and the use of different technologies can cause minor changes in the performance of the system from the complexity point of view. 
Table 2. Technologies of localization server and mobile station.

\begin{tabular}{ll}
\hline & Localization Server \\
\hline Hardware & Depends on performance requirements \\
Operating system & Microsoft Windows Server (latest) \\
Web server & Internet Information Services \\
Database & Microsoft SQL Server (latest) \\
Software platform & Microsoft .NET Framework \\
Programming language & C\# \\
\hline & Mobile Station \\
\hline \multirow{2}{*}{ Hardware } & Mobile phone with GNSS, GSM and Wi-Fi \\
& enabled hardware \\
Operating system & Android (2.3 and newer) \\
Programe platform & Android SDK \\
\hline
\end{tabular}

Localization server software is built on technologies from Microsoft because of our extensive experience with their implementation, but also due to the presence of high quality tools and a vast user and programmer documentation. Data storage uses a SQL-based relational database system and thus enables advanced data analysis with other tools directly from the data of the localization system.

The mobile station software is built on the Android operating system, which allows better access to the hardware equipment of the mobile station compared to other mobile operating systems such as Apple iOS or Microsoft Windows Phone. Furthermore the Java programming language is up to certain level similar to $\mathrm{C \#}$ language that has been used in the localization server.

The data transfer between a mobile station and the localization server is based on Hypertext Transfer Protocol (HTTP) and underlying TCP/IP protocol suite. The advantage of the HTTP protocol is in its wide use, therefore it is enabled on the communication networks, devices, operating systems and it passes network firewalls easily. Efficiency of the communication is achieved by Representational State Transfer (REST) principles used to expose localization server web services. The REST architecture represents a lightweight, computational and data transfer efficient alternative to the Simple Object Access Protocol (SOAP), which has been used for web services for years. The REST architecture saves computational resources on the mobile stations, where it is highly demanded as well as on the localization server.

Another benefit of the HTTP protocol is its integrated support for authentication and data encryption. Users are authenticated via basic HTTP authentication. Transmitted data are encrypted with Transport Layer Security (TLS) or Secure Sockets Layer (SSL) protocols.

\subsubsection{User View}

User view represents the capabilities of the system from the user perspective. Users can be divided into groups, according to their role and permissions. Basic user groups are:

- Standard user-Uses the system to obtain localization information.

- Installer-Sets up the system before it can be used by standard users and administrators.

- Administrator-Monitors system operation, updates the system and solves problems. 
A person can be member of more than one group, for instance if the system administrator needs to obtain localization information, he would become standard user as well. Each action in the localization system requires membership in certain set of groups as shown in Table 3 below.

Table 3. Actions in the localization system and user groups allowed performing them.

\begin{tabular}{ll}
\hline Action (Permission) & Allowed Groups \\
\hline Localize own person & Standard users \\
Localize someone else & - \\
Track own person & Standard users \\
Fill the database with necessary data & Installer, Administrator \\
Monitor the system & Administrator \\
Manage administrators & Installer \\
Manage users & Administrator \\
\hline
\end{tabular}

There are necessary steps to set up and configure the localization systems before it can provide the localization service. Three phases of the localization system lifecycle have been identified, namely:

- Installation - Installer configures the localization server and creates one or more system administrators.

- Initialization-Administrator creates accounts for users of services and installer fills the database with auxiliary data for localization modules such as fingerprinting radio map.

- Normal operation of the system-Standard users utilize localization service, the administrator monitors statistics and resolves any problems in the system. Administrator also updates the database with new auxiliary data.

\subsection{Map Reduction Algorithm}

In a modular localization system the area where the positioning services will be provided is significantly larger, thus the size of radio map is increased. This may result in a significant increase of the time required for the localization server to estimate the position of a mobile device. Another reason to optimize the complexity of the positioning process is the ability of the localization server to provide responses to localization requests from a large number of devices.

The aforementioned facts were the motivation to modify basic algorithms in order to reduce the complexity of the positioning process. The idea of the proposed algorithm is to filter out vectors that contain measurements from the same transmitters as the mobile device detected during the positioning process. In this way we can determine which vectors are in the area of mobile device and use them in the position estimation process.

We called the proposed optimization algorithm as a 2-phase map reduction algorithm. In the first phase only relevant areas are selected from the radio map database. In the second phase reference points are selected from the relevant areas, thus the complexity of the system is reduced even more significantly. A flowchart of the proposed algorithm in comparison with the basic algorithm is depicted in Figure 5. The first phase of the proposed algorithm retrieves all vectors that match at least one transmitter with the measurement from mobile device. This is easily preformed via SQL language and the initial filtering reduces the radio map to the areas with least one matching transmitter in the range. 
Figure 5. Flowcharts of the basic fingerprinting (a) and fingerprinting with 2-phase map reduction algorithm (b).

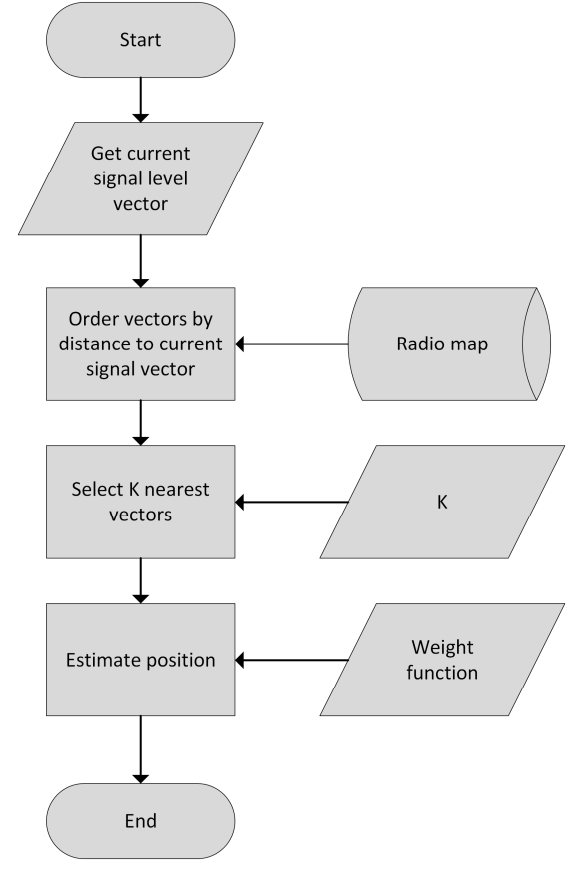

(a)

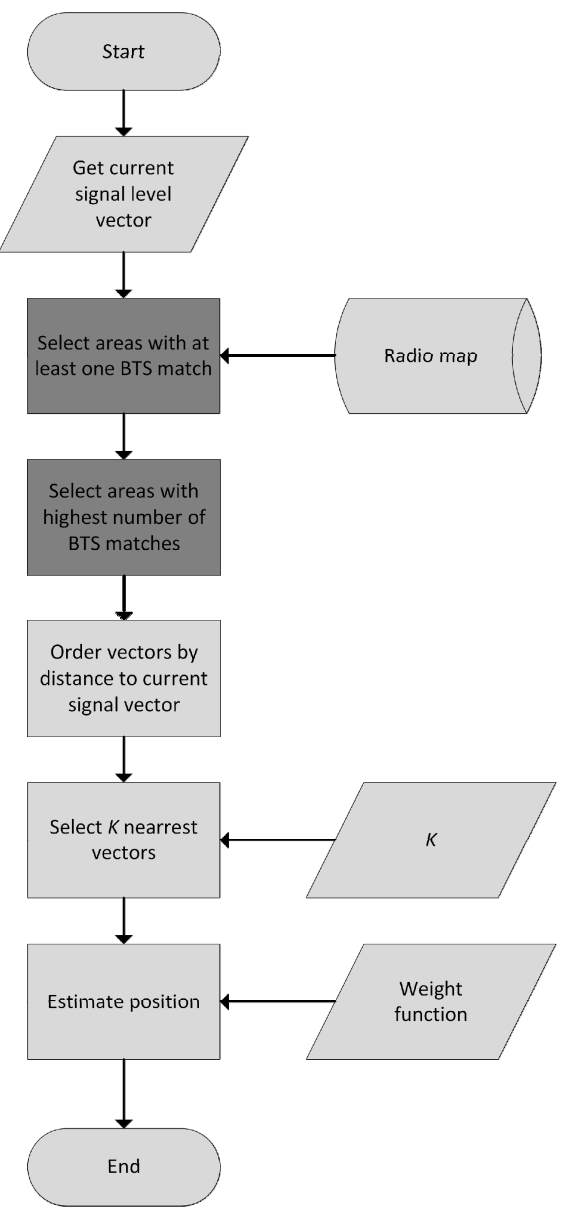

(b)

Performance of this step can be improved by sound database administration practices, such as indexing or partitioning, which can pay off when the number of reference points in the map reaches millions. This reduction of the radio map is shown in the Figure 6. The algorithm will be described using a simple example, where only three transmitters (BTS1, BTS2 and BTS3) were detected during the offline phase and their signals are stored in the radio map. On the other hand, the mobile device detects signals from only two of them (BTS2 and BTS3) during the positioning phase.

In Figure 6 the situation when the mobile device did not detect signals from BTS1 during the positioning phase is depicted. Areas chosen by the first phase of the algorithm are marked with a diagonal pattern. It can be seen that areas where signals from BTS1 should be detected were also chosen. It can be stated that in the first phase of the algorithm, reference points were chosen from area covered by signals from at least one transmitter detected in the radio map, other than BTS1.

In the second part of the proposed map reduction algorithm, it selects the most appropriate areas from the relevant areas chosen during the first stage as can be seen in Figure 7. This step is performed by ordering all areas by number of transmitters in the radio map vectors that match the transmitters detected by mobile device during the positioning phase. 
Figure 6. Phase 1 of map reduction algorithm.

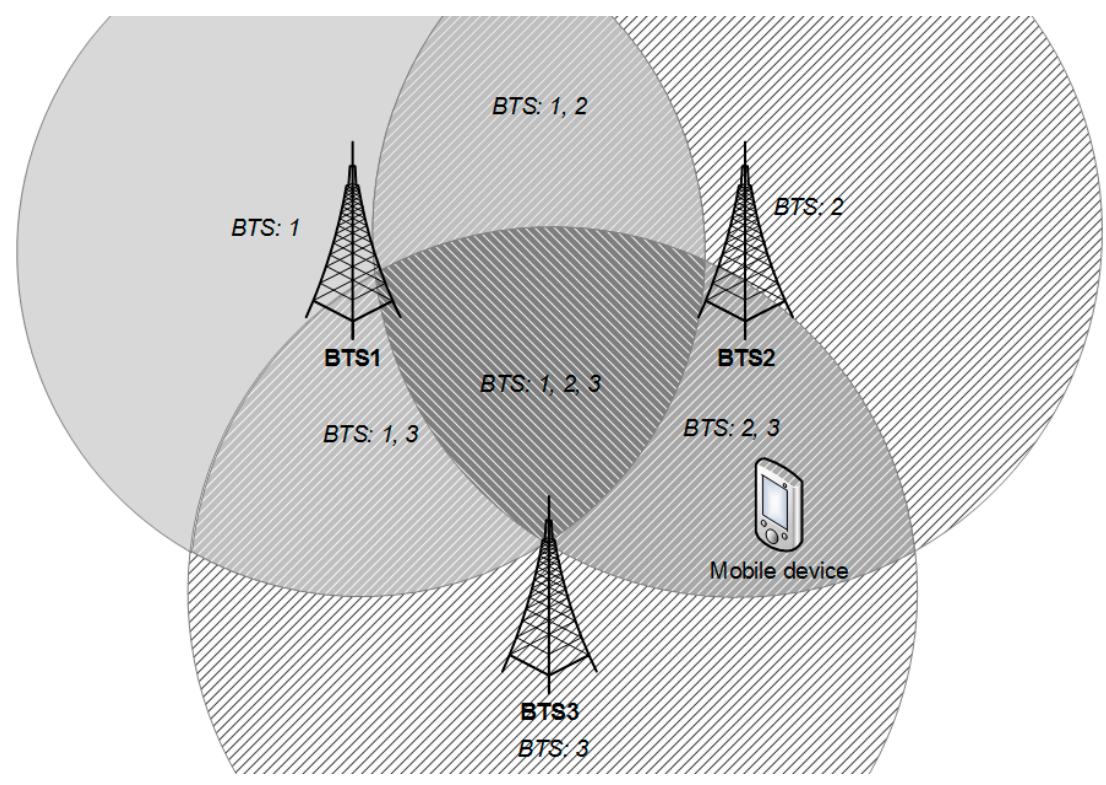

Figure 7. Phase 2 of map reduction algorithm.

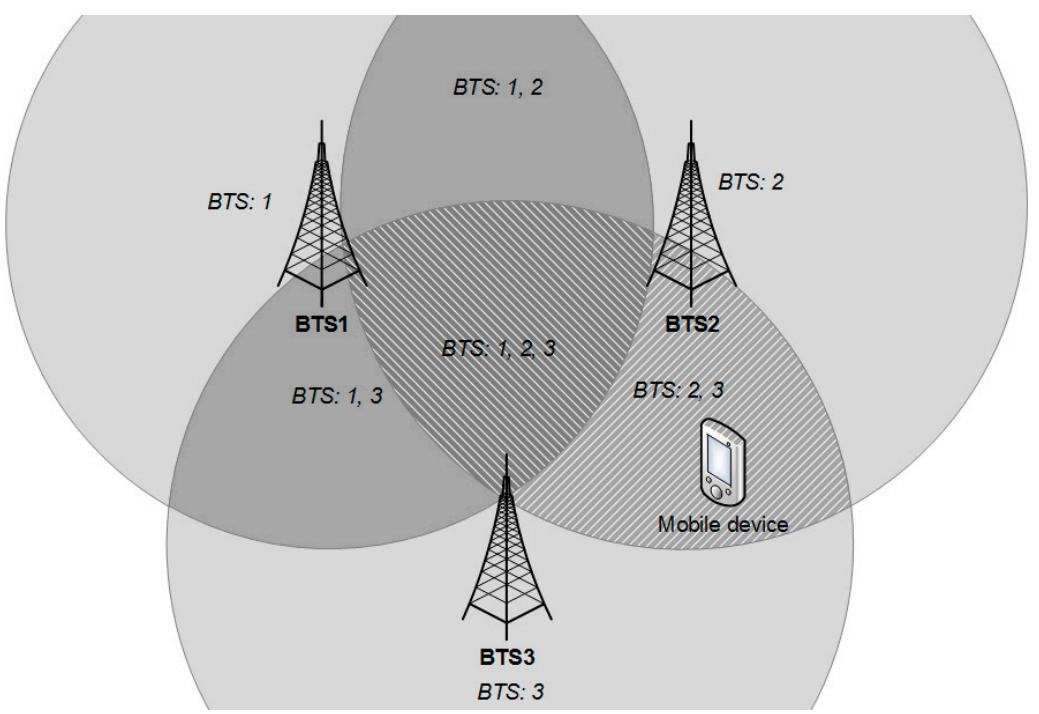

As stated before, only signals from transmitters BTS2 and BTS3 were detected during the positioning phase. Therefore the highest number of matches is 2 and reference points were chosen only from two small areas, where signals from both transmitters can be detected. The highest number of matches is selected in order to handle power fluctuations which may cause the measurement vector from the mobile device to contain more or less transmitters that were detected at the reference points in the radio map database.

\section{Testing Methodology and Setup}

Measurements were performed in the real world environment without any changes in the network infrastructure. Measurements were performed using implementation of the proposed modular localization system. The measurements were always coupled with the actual (i.e., real) position coordinates either to create the radio map or during the positioning of mobile device, to compare 
estimated and actual positions. The actual position was taken from GPS in the outdoor environment. In the indoor environment actual position was entered manually in internal building coordinate system. Measurements in both phases were performed using Sony Xperia Arc with Android OS version 4.0 and the server software was installed on an Acer TravelMate 5744 equipped with Microsoft Windows 8.1. Both indoor and outdoor environments contained radio maps created from Wi-Fi as well as GSM. The RSS measurements in radio map use signal averaging to reduce fluctuations and improve robustness of the positioning system. Average RSS value was calculated as:

$$
\overline{R S S}=\frac{1}{N_{s}} \sum_{i=1}^{N_{S}} R S S_{i}
$$

where $N_{S}$ is the number of samples, in the measurements set to 5, and $R S S_{i}$ represents $i$-th RSS sample. Radio maps were created during the evening hours, when the amount of traffic and pedestrians was lower, to achieve optimal results according to [26]. Measurements were performed in both indoor and outdoor environments. Radio maps for Wi-Fi and GSM in the outdoor environment can be seen in Figure 8.

Figure 8. Outdoor radio maps for (a) GSM and (b) Wi-Fi displayed on Open Street Map.

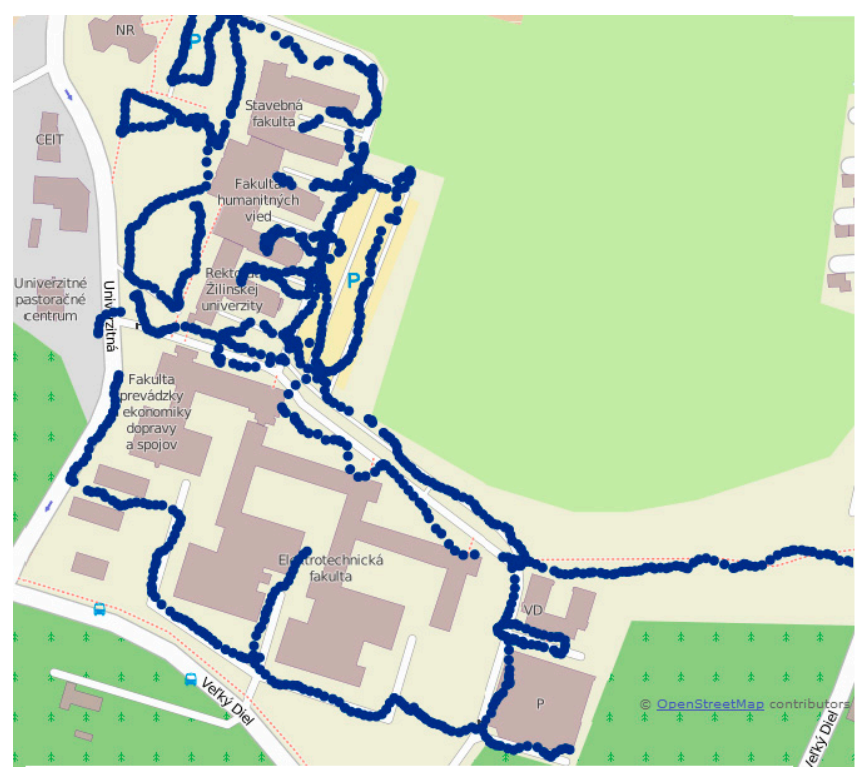

(a)

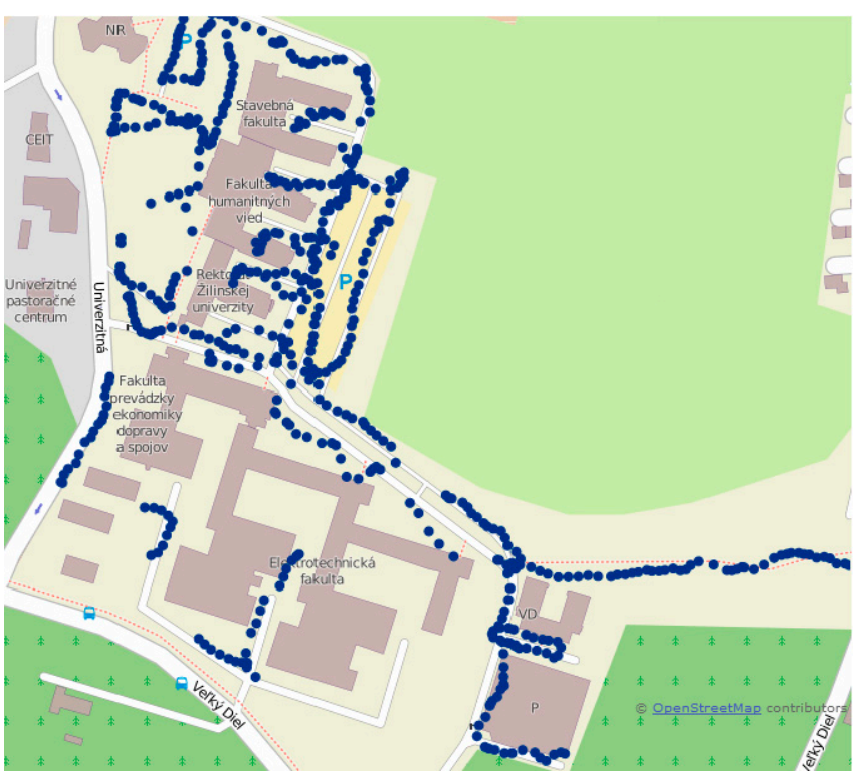

(b)

Measurements in the outdoor environment were performed on the campus of University of Zilina; the size of the area was $780 \times 470 \mathrm{~m}$. The GSM radio map was created with 937 reference points. On average five BTS were detected per reference point. GSM measurements were performed in a single network operated by Telefonica Slovakia. On the other hand, the radio map created using Wi-Fi signals contained 574 reference points. This difference is given by the fact that reference points with less than three APs detected by the device were excluded from the radio map database. On average 15 APs were detected per reference point.

The indoor measurements were performed in an office building with brick walls located in Zilina. The size of the area was $15 \times 8 \mathrm{~m}$. The GSM radio map consists of 50 reference points. At reference points the average number of detected signals from BTS stations was 4.5. The spatial distribution of reference points in the indoor environment for both Wi-Fi and GSM is shown in Figure 9. 
Figure 9. Indoor radio maps for (a) GSM and (b) Wi-Fi displayed on a plan of the building.

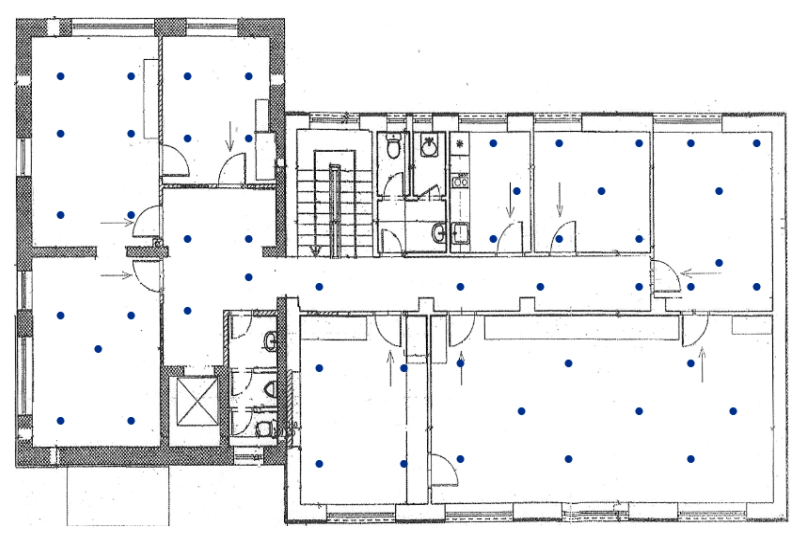

(a)

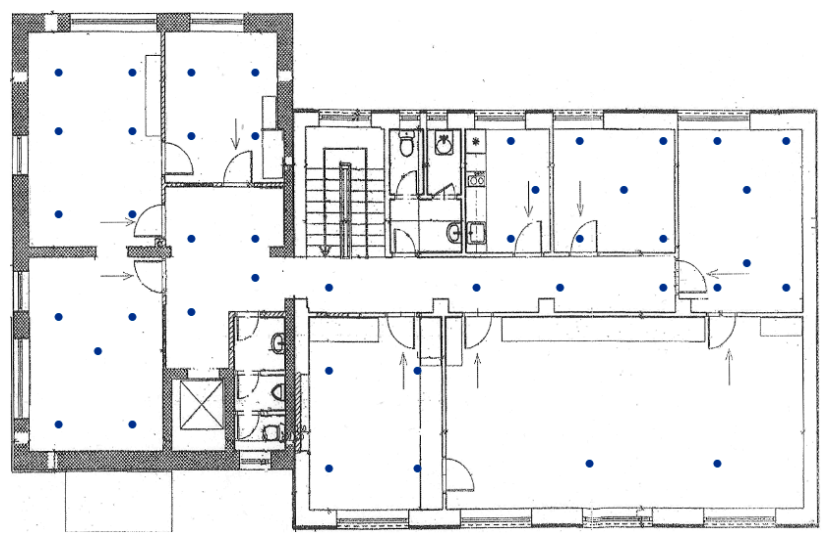

(b)

The radio map created using Wi-Fi consists of 43 reference points with average of more than 4 APs detected per reference point. Like the outdoor environment, the Wi-Fi radio map consists of a lower number of reference points compared to the GSM radio map, because a lower number of APs was in the range compared to the outdoor environment.

In the next section results achieved in the real world measurements will be presented and discussed. In order to do that we need to define the accuracy measures which were used to evaluate performance of the proposed positioning system. Accuracy is commonly expressed by the value of localization error, which is in fact a random variable and thus should be evaluated by statistical functions. Whereas accuracy usually refers to mean (i.e., average) error values, the term precision is used for localization error-related properties.

The accuracy is commonly evaluated by Mean Square Error (MSE), Root Mean Square Error (RMSE), Circular Error Probability (CEP) and Cumulative Distribution Function (CDF). The MSE, in localization also referred to as Mean Distance Error (MDE), expresses average of the squares of localization error, then RMSE represents average localization error. Using 2D coordinates, the values can be calculated as:

$$
\begin{gathered}
M S E=\frac{1}{N} \sum_{i=1}^{N}\left[\left(x_{i}-\hat{x}_{i}\right)^{2}+\left(y_{i}-\hat{y}_{i}\right)^{2}\right] \\
R M S E=\sqrt{M S E}
\end{gathered}
$$

where $\left[x_{i} ; y_{i}\right]$ are actual position coordinates of mobile device in $i$-th positioning step, $\left[\hat{x}_{i} ; \hat{y}_{i}\right]$ are coordinates of estimated position, $M$ is number of measurements. The lower the values of MSE and RMSE, the higher the accuracy of position estimates. These metrics can be used to evaluate $2 \mathrm{D}$ as well as $3 \mathrm{D}$ accuracy. In the result section, every RMSE value is accompanied with standard deviation $\sigma$ of values in a form $\mathrm{RMSE} \pm \sigma$.

The CEP represents the radius of the circle where $50 \%$ of the estimated positions have an error lower than or equal to the accuracy value. The lower the value of CEP, the higher the accuracy of position estimates. This metric can be used to evaluate 2D accuracy. Similar circles can be defined for 
other statistically interesting values such as 67\% (R67) or 95\% (R95). R95 is one of the most important metrics to decide which of the two systems is more precise.

The CDF describes the probability that localization error will be smaller than some pivot value V. For $\mathrm{N}$ measurements in the set, it can be calculated as:

$$
C D F(V)=\frac{1}{N(R M S E<V)}
$$

CDF is commonly expressed by a function graph from starting from $V=0$ and ending where $\mathrm{CDF}(\mathrm{V})$ is close to 1 . The metric can be used to evaluate both $2 \mathrm{D}$ and $3 \mathrm{D}$ accuracy.

Testing measurements were performed in two scenarios. The first scenario was proposed to evaluate impact of the proposed algorithm for map reduction. In this scenario outdoor positioning was performed with both Wi-Fi and GSM networks separately. During the measurements the localization accuracy and server response times were monitored.

The second scenario was proposed to evaluate performance of MLA algorithm in both indoor and outdoor environments. In the measurements localization accuracy of the modular localization system was monitored. In the measurements GPS module was not considered in the modular system. This should simulate the worst case scenario when GPS is not available and helps us to evaluate the performance of backup modules.

\section{Achieved Results and Discussion}

In the first scenario impact of the proposed 2-phase algorithm for map reduction on the performance of positioning based on fingerprinting framework was tested. The implemented algorithm was verified in the outdoor environment with both GSM and Wi-Fi radio maps. One hundred measurements were performed for each position estimator and network. The accuracy results before and after application of the proposed algorithm improvements are shown in Table 4.

From the results in the table it is obvious that map reduction improves the accuracy even if it was initially designed to only improve the performance. It can be seen that the mean localization error was reduced by $18 \mathrm{~m}$ for $\mathrm{KNN}$, by $35 \mathrm{~m}$ for WKNN and approximately by $55 \mathrm{~m}$ for NN algorithm when GSM signals were used to estimate the position. On the other hand, improvement for Wi-Fi based positioning is even more significant. It can be seen that mean localization error was reduced from approximately $180 \mathrm{~m}$ to only $20 \mathrm{~m}$, which is a significant improvement. This improvement is caused by the inaccuracy of the basic NN family algorithms, given by the fact that these algorithms do not consider the number of transmitters used to calculate the distance between RSS vectors. Thus basic algorithms can select RPs with only one detected transmitter with similar power, however in a completely different area, since other transmitters present at the given RP were not detected by the mobile device, the algorithms do not penalize this RP for missing transmitters and if the difference of RSS is relatively low, the position estimate is far from the real position. This problem of NN family algorithms emerges only when positioning is performed in large areas, since in small areas (e.g., buildings) the same transmitters are detected on all RPs. 
Table 4. Impact of map reduction on localization accuracy.

\begin{tabular}{cccc}
\hline \multirow{2}{*}{ Network } & Estimator & Basic Algorithm & With Map Reduction \\
\cline { 3 - 4 } & & RMSE [m] & RMSE [m] \\
\hline \multirow{3}{*}{ GSM } & $N N$ & $144.78 \pm 78.15$ & $89.67 \pm 64.61$ \\
& $K N N$ & $110.44 \pm 71.02$ & $92.42 \pm 50.90$ \\
& $W K N N$ & $120.63 \pm 69.18$ & $85.10 \pm 61.45$ \\
\hline \multirow{3}{*}{ Wi-Fi } & $N N$ & $178.47 \pm 47.57$ & $18.20 \pm 10.74$ \\
& $K N N$ & $181.24 \pm 29.60$ & $21.66 \pm 13.18$ \\
& $W K N N$ & $181.42 \pm 29.60$ & $19.22 \pm 8.70$ \\
\hline
\end{tabular}

The impact of the proposed 2-phase map reduction algorithm on performance of the localization algorithm is shown in Table 5. In the table $T$ represents time of basic algorithm and $T_{M R}$ represents time after application of the map reduction algorithm. In the performance testing two kinds of different positioning requests, i.e., valid requests and invalid requests, were used. Valid requests mean that measurement vector consists of RSS measurements from transmitters present in the radio map database and therefore the position of the mobile device can be estimated. On the other hand, invalid requests were generated with transmitters that were not present in the radio map database, meaning the measurements were performed in the area where the positioning service is not available and the position of a mobile device cannot be estimated.

Table 5. Impact of proposed map reduction algorithm on the response time of localization server.

\begin{tabular}{lcclcc}
\hline \multirow{2}{*}{ Metric } & \multicolumn{2}{c}{ GSM } & & \multicolumn{2}{c}{ Wi-Fi } \\
\cline { 2 - 3 } \cline { 5 - 6 } & $\boldsymbol{T}[\mathbf{s}]$ & $\boldsymbol{T}_{\boldsymbol{M R}}[\mathbf{s}]$ & & $\boldsymbol{T}[\mathbf{s}]$ & $\boldsymbol{T}_{\boldsymbol{M R}}[\mathbf{s}]$ \\
\hline Single valid request & $0.68 \pm 0.06$ & $0.55 \pm 0.17$ & & $4.31 \pm 2.13$ & $0.76 \pm 0.60$ \\
10 parallel valid requests & $3.44 \pm 0.37$ & $2.84 \pm 0.95$ & & $23.14 \pm 11.34$ & $3.96 \pm 3.33$ \\
100 parallel valid requests & $30.76 \pm 8.87$ & $25.79 \pm 10.81$ & & $289.86 \pm 147.66$ & $45.79 \pm 41.90$ \\
Single invalid request & $0.86 \pm 0.14$ & $0.006 \pm 0.001$ & & $4.49 \pm 2.37$ & $0.014 \pm 0.007$ \\
10 parallel invalid requests & $4.32 \pm 0.66$ & $0.017 \pm 0.006$ & & $24.15 \pm 12.22$ & $0.053 \pm 0.033$ \\
100 parallel invalid requests & $39.15 \pm 12.27$ & $0.220 \pm 0.085$ & & $308.78 \pm 178.89$ & $0.505 \pm 0.327$ \\
\hline
\end{tabular}

It can be seen that map reduction significantly reduces the duration of the localization algorithm. The positive effect is more obvious in the Wi-Fi radio map, since Wi-Fi fingerprint vectors contain more transmitters thus the radio map is bigger. Another positive effect of map reduction is that it reduces the duration by three orders of magnitude for invalid localization requests.

The high values of standard deviation result from the fact that the application was running on a computer running on Windows OS and the computation time was affected by other processes running in the background, which causes high differences in computation times. It can be seen that the time needed to perform Wi-Fi-based positioning was significantly higher compared to the time needed to estimate the position using GSM signals. This was given by the fact that in Wi-Fi a higher number of transmitters was detected. Therefore more computations need to be performed to calculate the distances between RSS vectors.

In the last scenario, the positioning was performed in both indoor and outdoor environments at 10 evenly distributed positions. There were 10 localization requests executed at each position for all implemented positioning algorithms- $\mathrm{NN}, \mathrm{KNN}$ and WKNN. Altogether there were 100 position 
estimations performed by each of the algorithms and environment. The achieved accuracy of the modular positioning system is shown in Tables 6 and 7 for indoor and outdoor environments, respectively.

Table 6. Accuracy of the modular positioning system in an outdoor environment.

\begin{tabular}{ccccc}
\hline Estimator & RMSE $[\mathbf{m}]$ & CEP $[\mathbf{m}]$ & $\mathbf{6 7 \%}[\mathbf{m}]$ & $\mathbf{9 5 \%}[\mathbf{m}]$ \\
\hline NN & $22.58 \pm 23.20$ & 16.60 & 23.44 & 71.93 \\
KNN & $36.20 \pm 33.82$ & 22.37 & 31.68 & 116.52 \\
WKNN & $25.38 \pm 22.04$ & 19.30 & 22.76 & 81.06 \\
\hline
\end{tabular}

Table 7. Accuracy of modular positioning system in the indoor environment.

\begin{tabular}{ccccc}
\hline Estimator & RMSE $[\mathbf{m}]$ & CEP $[\mathbf{m}]$ & $\mathbf{6 7 \%}[\mathbf{m}]$ & $\mathbf{9 5 \%}[\mathbf{m}]$ \\
\hline NN & $2.81 \pm 2.65$ & 2.15 & 3.58 & 7.35 \\
KNN & $2.58 \pm 1.95$ & 1.73 & 3.97 & 5.84 \\
WKNN & $2.74 \pm 2.13$ & 1.91 & 3.97 & 6.87 \\
\hline
\end{tabular}

From the achieved results it can be seen that positioning accuracy achieved by MLA positioning is slightly lower compared to the accuracy achieved by the Wi-Fi positioning with map reduction. However it is important to notice that Wi-Fi positioning is not available all the time and a low number of transmitters in the range can result in invalid positioning requests. Thus the accuracy of MLA is affected by GSM position estimates, which cannot achieve the accuracy of Wi-Fi positioning. In the outdoor environment the MLA selected Wi-Fi localization instead of GSM in 78\% of cases, whereas GSM has been used only in $22 \%$ of cases. This is mostly caused by the signal coverage, because there were fewer positions with poor or no Wi-Fi coverage. For the better analysis of the achieved results CDF of localization error achieved by MLA in outdoor environment is shown in Figure 10.

Figure 10. CDF of localization error in the outdoor environment.

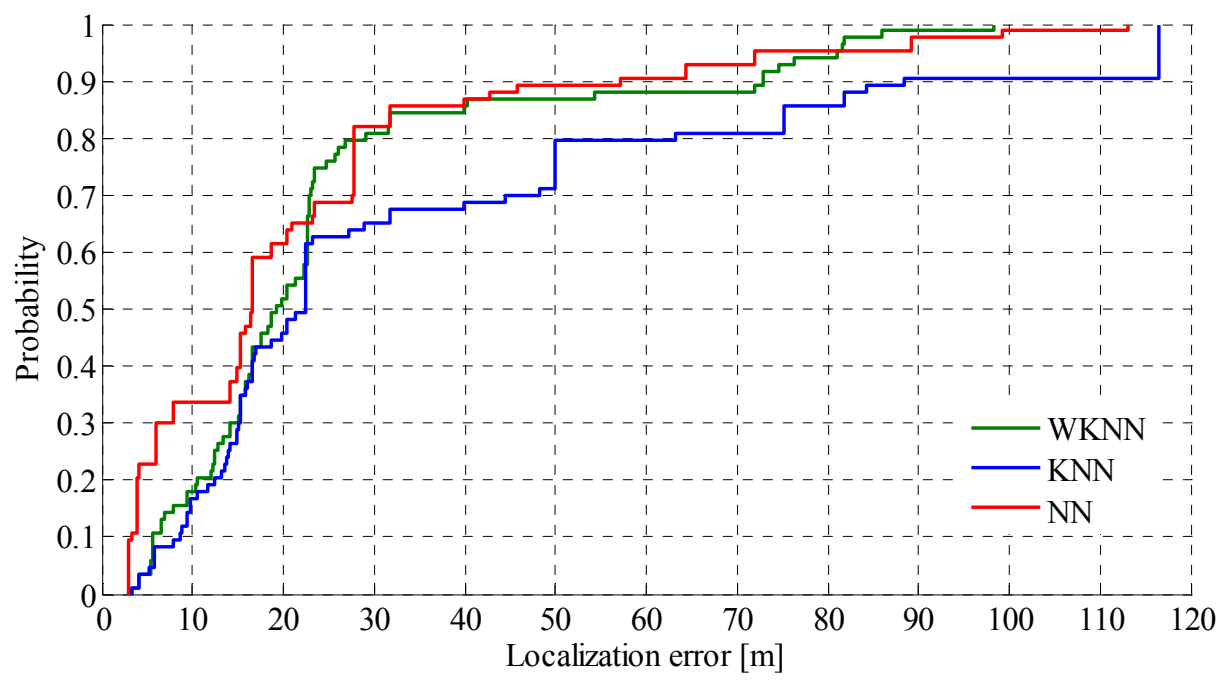

From the figure it can be seen that $\mathrm{NN}$ algorithm achieved the best results and is able to estimate the position of mobile devices with an accuracy of less than $20 \mathrm{~m}$ in more than $60 \%$ of requests. It can also be seen that worst results were achieved by the KNN algorithm. 
In Table 7, the achieved results for an indoor environment are depicted. There it can be seen that the accuracy achieved with the MLA algorithm is much better compared to the outdoor environment. This is caused by the fact that radio signals are attenuated by the walls and furniture in the building, which results in the higher differences in RSS measured at reference points. In the indoor environment the MLA preferred Wi-Fi localization over GSM in 51\% of cases. This is caused by the signal propagation in the environment, and it shows that in $49 \%$ of cases, there were less than three Wi-Fi APs with RSS greater than $-90 \mathrm{dBm}$ in the range of mobile device. Like the outdoor environment, the results achieved for the indoor environment are shown as CDF of localization error in Figure 11.

Figure 11. CDF of localization error in indoor environment.

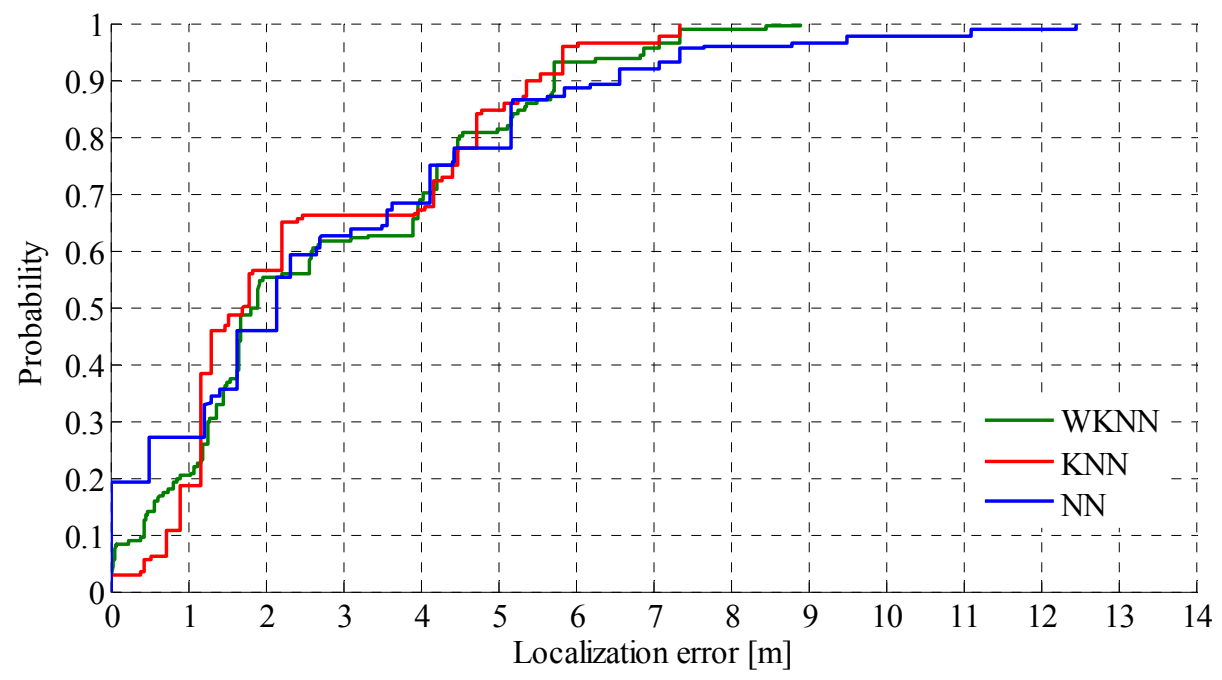

From the results shown in the figure it can be seen that, in contrast to the outdoor environment, in the indoor environment positioning using the KNN algorithm achieved best results. It can be seen that more than $55 \%$ of position estimates have errors lower than $2 \mathrm{~m}$, which is a good result.

The differences between particular estimators are not significant; therefore we decided to implement $\mathrm{NN}$ in our modular localization system. The next very important fact is the computational capacity of particular estimators. The NN requires the lowest computational capacity therefore the decision seems to be optimal.

\section{Conclusions and Future Work}

In this paper a novel 2-phase map reduction algorithm and modular localization were proposed. Both algorithms were implemented in a localization system to evaluate their impact on the performance of the positioning system developed at the University of Zilina. Implemented algorithms were tested in real world conditions in both indoor and outdoor environments. From the achieved results it can be seen that proposed 2-phase map reduction algorithm reduced the complexity of the positioning process and also improved the accuracy of the position estimates. It has to be noted that the proposed algorithm allows detecting and ignoring false positioning requests, and thus can significantly reduce complexity.

A modular positioning systems that can utilize Wi-Fi and GSM signals was proposed and developed. The main contribution is the proposal of the modular localization algorithm, which is able 
to automatically switch between different localization modules. During the test, GPS positioning was disabled in the MLA algorithm, to determine performance of the positioning modules based on radio networks. The achieved mean localization error was $20 \mathrm{~m}$ in the outdoor environment and less than $3 \mathrm{~m}$ in the indoor environment. From the results it is obvious that positioning in outdoor environments still needs to be improved. However it must be noted that measurements were not performed in dense urban area, where a larger number of wireless transmitters and more buildings are present. This could allow slightly higher positioning accuracy. The proposed system can be used in transport for navigation purposes, since it can operate in both indoor and outdoor environments seamlessly.

In the future research the focus should be put mainly on optimization of the proposed positioning system, especially in outdoor environments, in order to provide more accurate results, even when GNSS-based positioning is not possible. We will also focus on development of an algorithm which will provide more precise switching between outdoor and indoor environments. Another interesting topic for the future research that can be performed on the proposed modular system is the impact of using different devices on the accuracy of the system.

\section{Acknowledgments}

This work was supported by the Slovak VEGA grant agency, Project No. 1/0394/13.

\section{Author Contributions}

All of the authors have significantly contributed to the research. Jozef Benikovsky contributed to proposal of algorithms, software implementation and data collection. Peter Brida has provided guidance during the whole research. Juraj Machaj contributed by organizing results and the manuscript.

\section{Conflicts of Interest}

The authors declare no conflict of interest.

\section{References}

1. Mohapatra, D.; Suma, S.B. Survey of location based wireless services. In Proceedings of the IEEE International Conference Personal Wireless Communications 2005, ICPWC 2005, New Delhi, India, 23-25 January 2005; pp. 358-362.

2. Cerny, M.; Penhaker, M. Wireless body sensor network in health maintenance systems. Electron. Electr. Eng. 2011, 115, 113-116.

3. Mautz, R. The challenges of indoor environments and specification on some alternative positioning systems. In Proceedings of the 6th Workshop on Positioning, Navigation and Communication 2009, WPNC 2009, Hannover, Germany, 19 March 2009; pp. 29-36.

4. Zheng, L.; Dehaene, W.; Gielen, G. A 3-Tier UWB-based indoor localization scheme for ultra-low-power sensor nodes. In Proceedings of the IEEE International Conference on Signal Processing and Communications, ICSPC 2007, Dubai, 24-27 November 2007; pp. 995-998. 
5. Xing, B.; Geng, Z.; Han, L.; Du, S. Intelligent Alarm Positioning System Based on Zigbee Wireless Networks. In Proceedings of the 6th International Conference on Intelligent Networks and Intelligent Systems (ICINIS), Shenyang, China, 1-3 November 2013; pp. 278-281.

6. Chawathe, S. Low-latency indoor localization using Bluetooth beacons. In Proceedings of the 12th International IEEE Conference on Intelligent Transportation Systems, ITSC'09, St. Louis, MO, USA, 4-7 October 2009; pp. 1-7.

7. Koski, L.; Perälä, T.; Piché, R. Indoor positioning using WLAN coverage area estimates. In Proceedings of the 2010 International Conference on Indoor Positioning and Indoor Navigation, IPIN2010, Zurich, Switzerland, 15-17 September 2010.

8. Behan, M.; Krejcar, O. Modern smart device-based concepr of sensoric networks. Eurasip J. Wirel. Commun. Netw. 2013, 155, doi:10.1186/1687-1499-2013-155.

9. Honkavirta, V.; Perälä, T.; Ali-Löytty, S.; Piché, R. A comparative survey of WLAN location fingerprinting methods. In Proceedings of the 6th Workshop on Positioning, Navigation and Communication, WPNC 2009, Hannover, Germany, 19 March 2009; pp. 243-251.

10. Li, B.; Salter, J.; Dempster, A.G.; Rizos, C. Indoor Positioning Techniques Based on Wireless $L A N$; Tech. Report; School of Surveying and Spatial Information Systems, UNSW: Sydney, Australia, 2006.

11. Saha, S.; Chauhuri, K.; Sanghi, D.; Bhagwat, P. Location determination of a mobile device using IEEE $802.11 \mathrm{~b}$ access point signals. In Proceedings of the Wireless Communications and Networking, WCNC 2003, New Orleans, LA, USA, 16-20 March 2003; Volume 3, pp. 1987-1992

12. Drane, C.; Macnaughtan, M.; Scott, C. Positioning GSM telephones. IEEE Commun. Mag. 1998, $36,46-54$.

13. Gustafsson, F.; Gunnarsson, F. Mobile Positioning Using Wireless Networks. IEEE Signal Process. Mag. 2005, 22, 41-53.

14. Shen, J.; Oda, Y. Direction Estimation for Cellular Enhanced Cell-ID Positioning Using Multiple Sector Observations. In Proceedings of the 2010 International Conference on Indoor Positioning and Indoor Navigation (IPIN), Zurich, Switzerland, 2010.

15. Varshavsky, A.; de Lara, E.; LaMarca, A.; Hightower, J.; Otsason, V. GSM Indoor Localization. Pervasive Mob. Comput. J. (PMC) 2007, 3, 698-720.

16. Cipov, V.; Dobos, L.; Papaj, J. ToA Node Distance Estimation Enhancement in MANET Localization Algorithm Based on Cooperative Trilateration. Adv. Electr. Electron. Eng. 2012, 10, 211-217.

17. Wong, C.; Klukas, R.; Messier, G. Using WLAN Infrastructure for Angle-of-Arrival Indoor User Location. In Proceedings of the IEEE Vehicular Technology Conference, VTC 2008-Fall, Calgary, BC, Canada, 21-24 September 2008.

18. Muthukrishnan, K.; Koprinkov, G.; Meratnia, N.; Lijding, M. Using Time-of-Flight for WLAN Localization: Feasibility Study; Technical Report No. TR-CTIT-06-28; Centre for Telematics and Information Technology (CTIT), University of Twente: Enschede, Netherlands, 2006.

19. Golden, S.; Bateman, S. Sensor Measurements for Wi-Fi Location with Emphasis on Time-of-Arrival Ranging. IEEE Trans. Mob. Comput. 2007, 6, 1185-1198. 
20. Günther A.; Hoene, C. Measuring Round Trip Times to Determine the Distance between WLAN Nodes; Technical Report No. TKN-04-16; Telecommunication Networks Group, Technical University Berlin: Berlin, Germany, 2004; p. 43.

21. Mazuelas, S.; Bahillo, A.; Lorenzo, R.M.; Fernandez, P.; Lago, F.A.; Garcia, E.; Blas, J.; Abril, J. Robust Indoor Positioning Provided by Real-Time RSSI Values in Unmodified WLAN Networks. IEEE J. Sel. Top. Signal Process. 2009, 3, 821-831.

22. Bahl, P.; Padnababhan, V.N. RADAR: An in-building RFbased user location and tracking system. In Proceedings of the Nineteenth Annual Joint Conference of the IEEE Computer and Communications Societies INFOCOM 2000, Tel Aviv, Israel, 26-30 March 2000.

23. Tsung-nan, L.; Po-Chiang, L. Performance comparison of indoor positioning techniques based on location fingerprinting in wireless networks. In Proceedings of the International Conference on Wireless Networks, Communications and Mobile Computing 2005, Maui, HI, USA, 13-16 June 2005; Volume 2.

24. Machaj, J.; Brida, P. Performance Comparison of Similarity Measurements for Database Correlation Localization Method. In Proceedings of the 3rd Asian Conference on Intelligent Information and Database Systems, ACIIDS 2011, Daegu, Korea, 20-22 April 2011; pp. 452-461.

25. Machaj, J.; Brida, P. Impact of Wi-Fi Access Points on performance of RBF localization algorithm. In Proceedings of the ELEKTRO, 2012, Rajeck Teplice, Slovakia, 21-22 May 2012; pp. 70-74.

26. Brida, P.; Machaj, J., Benikovsky, J. Effect of Environmental Changes on Accuracy of IEEE 802.11 Indoor Fingerprinting Positioning System WifiLOC. In Proceedings of the Abstract Volume of the 2010 International Conference on Indoor Positioning and Indoor Navigation (IPIN), Zurich, Switzerland, 27-30 October 2010; pp. 75-76.

(C) 2014 by the authors; licensee MDPI, Basel, Switzerland. This article is an open access article distributed under the terms and conditions of the Creative Commons Attribution license (http://creativecommons.org/licenses/by/4.0/). 